\title{
INTERVENÇÃO FONOLÓGICA EM CRIANÇAS \\ COM DISTÚRBIO ESPECÍFICO DE LINGUAGEM \\ COM BASE EM UM MODELO PSICOLINGUÍSTICO
}

\author{
Phonological intervention for children with \\ specific language impairment within a psycholinguistic model
}

Dáphine Luciana Costa Gahyva( ${ }^{(1)}$, Simone Rocha de Vasconcellos Hage ${ }^{(2)}$

\begin{abstract}
RESUMO
Tema: intervenção fonológica em crianças com Distúrbio Específico de Linguagem, com base em um Modelo Psicolinguístico. Procedimentos: participaram do processo de intervenção 4 pré-escolares, de ambos os gêneros, com idade entre 48 e 83 meses. O desempenho psicolinguístico dos sujeitos foi obtido em duas etapas (pré e pós-intervenção), mediante a utilização de instrumentos que avaliam diferentes níveis do processamento da informação: reconhecimento auditivo-fonético, consciência fonológica, codificação/ produção fonológica, memória de trabalho e acesso lexical. 0 Programa de intervenção teve duração de 4 meses. Resultados: os pré-escolares apresentaram comprometimento em todos os níveis do processamento da informação avaliados (pré-intervenção), revelando que as dificuldades de organização fonológica estavam relacionadas a problemas no processamento receptivo e expressivo. Ao fim do Programa, todos apresentaram melhora no desempenho fonológico. Conclusão: o uso de procedimentos de avaliação que abordam os diferentes níveis de processamento possibilita a compreensão da natureza dos distúrbios de linguagem e permitem a programação de estratégias mais efetivas para as dificuldades de linguagem.
\end{abstract}

DESCRITORES: Transtornos do Desenvolvimento da Linguagem; Testes de Linguagem; Terapia da Linguagem

\section{INTRODUÇÃO}

Ao longo dos anos, a literatura especializada tem demonstrado um grande interesse em pesquisas relacionadas à intervenção fonoaudiológica nos distúrbios de linguagem. Programas de intervenção com base em diferentes orientações teóricas sobre o processo de aquisição e desenvolvimento de linguagem têm sido desenvolvidos, propostos e testados, trazendo contribuições não somente para a verificação da eficácia do(s) procedimento(s) de

(1) Fonoaudióloga; Prefeitura Municipal de Uberaba, MG; Mestre em Fonoaudiologia pela Faculdade de Odontología de Bauru da Universidade de São Paulo.

(2) Fonoaudióloga; Professora Doutora do Departamento de Fonoaudiologia da Faculdade de Odontología de Bauru da Universidade de São Paulo, FOB-USP, Bauru, SP; Doutora em Neurociências pela Universidade Estadual de Campinas.

Conflito de interesses: inexistente interesse, mas também para o conhecimento teórico sobre mecanismos envolvidos com a linguagem.

Se por um lado existem programas que enfocam diretamente a organização dos subsistemas linguísticos (fonologia, sintaxe, semântica e pragmática); por outro, há uma série de trabalhos mais recentes que vem salientando as relações entre o desenvolvimento cerebral, o linguístico e o das demais funções cognitivas envolvidas com esta função. Tais relações dizem respeito aos vários processos mentais que permitem a compreensão de um enunciado e antecedem $o$ ato de falar, mais especificamente, se referem ao funcionamento de falantes reais em atividades de percepção, compreensão, memória, produção e metalinguagem ${ }^{1-5}$.

Os autores que se interessaram pelo entendimento da linguagem seguindo essa perspectiva apoiaram-se, num momento, nas teorias linguísticas, e, em outro, nos métodos de análise das teorias psicológicas. Servindo-se assim, de conceitos 
e metodologias das duas disciplinas, a abordagem psicolinguística fundamentou-se e se desenvolveu, com o propósito de propiciar o estabelecimento de hipóteses sobre os mecanismos subjacentes ao desenvolvimento da linguagem - tanto em um funcionamento normal como num patológico - e de definir estratégias de intervenção que não sejam guiadas unicamente por uma observação de superfície ${ }^{1,6,7}$

O modelo psicolinguístico tem influenciado decisivamente, na última década, a forma de conceber, avaliar e afrontar a intervenção nas dificuldades de linguagem. É o que tem se revelado mais útil no campo dos transtornos de linguagem, já que tenta explicar a forma como os seres humanos processam a informação que chega aos seus sentidos, para acessar as palavras armazenadas em seu léxico e usar as representações mentais que codificam as informações em vários níveis. Permite assim, a compreensão da natureza dos distúrbios de linguagem ${ }^{1,7}$.

Considera que o papel do fonoaudiólogo consiste em determinar, mediante diagnóstico diferencial, qual é a causa da sintomatologia apresentada, quais são os fatores psicolinguísticos implicados (ou em que ponto da cadeia de processamento existe uma ruptura que esteja impedindo o desenvolvimento normal da linguagem) e, de acordo com eles, desenhar um programa de intervenção individualizado que possibilite eliminar não só a sintomatologia apresentada pela criança, mas também os processos psicolinguísticos que a produzem. Isso porque, os modelos consideram que por trás de distúrbio de linguagem pode existir mais de uma causa que o produza ${ }^{7,8}$.

Assim, para que sejam aplicados de forma adequada, é aconselhável que os clínicos reflitam sobre três fatores: inicialmente, que selecionem um modelo para guiar o processo de avaliação e intervenção. Dentro do processo de avaliação, é imprescindível que este seja desenvolvido de uma forma detalhada, que permita a descrição dos processos diretamente envolvidos com a linguagem, assim como a interação entre eles e os processos cognitivos subjacentes. No tocante ao processo de intervenção, este deve enfocar os níveis comprometidos previamente identificados, de modo a possibilitar que a hipótese inicial sobre a natureza do déficit de linguagem seja testada. Se for observado, o desenvolvimento dos aspectos previamente comprometidos sinaliza que a(s) hipótese(s) levantada(a) realmente condiz(em) com as habilidades e deficiências da criança. Caso contrário, significa que a hipótese inicial estava incorreta, que a intervenção não abordou efetivamente os aspectos comprometidos ou que o modelo selecionado requer um maior aprimoramento ${ }^{9}$.

As teorias do processamento da informação também têm gerado controvérsias nos últimos anos. Tem-se apontado que, mesmo que uma criança aprenda e domine perfeitamente a execução das tarefas de processamento básico, ela não vai necessariamente melhorar as suas dificuldades linguísticas. Todavia, mesmo com toda a corrente crítica, os programas desenvolvidos, seguindo essa orientação, têm alertado os fonoaudiólogos sobre a necessidade de se investigar e de integrar, em intervenção, alguns aspectos não considerados previamente, como o treinamento da discriminação auditiva ${ }^{10-12}$, consciência fonológica ${ }^{13-15}$, velocidade de processamento e memória de trabalho ${ }^{2,4,5}$.

O objetivo do presente estudo foi verificar a eficácia da intervenção fonológica - com base em um Modelo Psicolinguístico ${ }^{1}$ - em crianças com Distúrbio Específico de Linguagem (DEL).

\section{APRESENTAÇÃO DOS CASOS}

Participaram do programa de intervenção 4 préescolares com DEL misto, sem comprometimentos práxicos, de ambos os sexos, com idade entre 48 e 83 meses.

Os sujeitos foram selecionados dentre aqueles atendidos na Clínica de Diagnóstico Fonoaudiológico da Faculdade de Odontologia de Bauru, da Universidade de São Paulo - USP, que tiveram o diagnóstico de DEL feito por equipe interdisciplinar formada por fonoaudiólogos - das especialidades de Linguagem e Audiologia - e por profissional psicólogo. Apresentaram as seguintes características, considerando os critérios de inclusão já propostos na literatura especializada: performance linguística abaixo do esperado para a idade mental e cronológica, considerando-se a expressão e compreensão da linguagem oral; limiares auditivos dentro dos padrões de normalidade; ausência de problemas comportamentais e/ou emocionais; desempenho cognitivo de acordo com a normalidade, ou ainda, discrepância entre o desempenho das habilidades cognitivas verbais e não-verbais; ausência de sintomatologia neurológica clássica, como por exemplo, Paralisia Cerebral, Deficiência Mental, Afasia Infantil (adquirida).

Como critério de inclusão neste grupo, além dos anteriormente citados, inerentes ao diagnóstico de DEL, também se exigiu que a criança nunca tivesse sido submetida à intervenção fonoaudiológica e possuísse idade igual ou superior a quatro anos, de modo a garantir a persistência das alterações de linguagem. 
O desempenho psicolinguístico dos pré-escolares foi obtido em duas etapas, sendo uma avaliação pré-intervenção (Al), realizada imediatamente antes do início do processo terapêutico e uma pósintervenção (AF), imediatamente ao fim do mesmo.

Importante ressaltar que o desempenho psicolinguístico dos pré-escolares com DEL - na avaliação pré-intervenção - foi comparado ao de um grupo controle (GC) composto por 4 pré-escolares com desenvolvimento cognitivo, de linguagem e de audição normais para a idade cronológica, de acordo com os critérios estabelecidos na literatura e obtidos mediante triagem fonoaudiológica. As crianças de ambos os grupos foram pareadas seguindo o critério idade cronológica.

A execução das avaliações envolveu a utilização dos seguintes instrumentos, que avaliam diferentes níveis do processamento da informação, considerando o modelo de avaliação Neuropsicolinguístico ${ }^{1}$ :

1. Reconhecimento auditivo-fonético: foi utilizado o Teste de Discriminação Auditiva ${ }^{16}$.

2. Consciência/análise fonológica: utilizado o teste Perfil de Habilidades Fonológicas ${ }^{17}$.

3. Acesso lexical: utilizada a prova de vocabulário do ABFW - Teste de Linguagem Infantil ${ }^{18}$.

4. Verificação do inventário fonético: utilizada a prova de fonética do ABFW - Teste de Linguagem Infantil ${ }^{19}$. O levantamento do inventário fonético foi obtido mediante prova de repetição de palavras. Foram considerados como dominados e presentes no inventário os fonemas produzidos com mais de $75 \%$ de acerto, de acordo com as possibilidades de ocorrência propostas pelo teste.

5. Organização/produção fonológica: utilizada a prova de Fonologia do ABFW - Teste de Linguagem Infantil ${ }^{19}$.

6. Memória de curto prazo: utilizado o Subteste Memória Sequencial Auditiva do ITPA ${ }^{20}$. O desempenho dos pré-escolares, nas duas avaliações, refere-se à Idade Psicolinguística em meses, obtida a partir do escore bruto alcançado na aplicação do teste.

O programa de intervenção teve duração de 4 meses. Durante todo o período foram realizados dois atendimentos semanais e individuais, de 45 minutos cada.

Respeitando-se a importância de todos os componentes do Modelo Psicolinguístico 1, as estratégias implementadas neste programa de intervenção foram desenvolvidas com o intuito de possibilitar às crianças a realização de discriminação entre os sons da fala, promover o desenvolvimento da memória de trabalho, tornar as representações fonológicas das palavras mais precisas, torná-las conscientes dos aspectos estruturais e funcionais da língua (atividades de consciência fonológica, consciência da palavra), torná-las capazes de acessar as representações no léxico mental e de realizar a programação, planejamento e produção motora de forma satisfatória. Ou seja, visaram intervir junto aos níveis do processamento afetados em cada caso.

Para cada sujeito, os objetivos da intervenção foram pré-determinados, baseados na avaliação prévia e trabalhados em sessões estruturadas, guiadas prioritariamente pelo terapeuta, em que pouca ênfase foi dada aos contextos naturais. As estruturas de intervenção, ou melhor, o tempo destinado às atividades de recepção, organização, produção e memória variou, de acordo com as necessidades dos sujeitos e das respostas das crianças às estratégias implementadas.

Considerando a disposição vertical do Modelo e a hierarquização dos níveis, o Programa de intervenção abordou inicialmente os níveis mais periféricos do processamento da linguagem oral, que condicionam amplamente o desenvolvimento e/ou o funcionamento dos níveis mais centrais.

Este trabalho foi previamente aprovado pelo Comitê de Ética em Pesquisa da Faculdade de Odontologia de Bauru, da Universidade de São Paulo, processo no 109/2005, e todos os pais e/ ou responsáveis autorizaram a participação do(a) filho(a) na pesquisa, mediante assinatura de Termo de Consentimento Livre e Esclarecido, conforme Resolução 196/96-CNS/MS.

Devido à pequena amostra constituinte deste estudo, os resultados serão apresentados de forma descritiva, baseados nas pontuações obtidas pelos sujeitos e médias apresentadas pelos grupos, nas diversas dimensões psicolinguísticas avaliadas.

\section{RESULTADOS}

Todas as crianças do GC apresentaram desempenho de acordo com os critérios de normalidade estabelecidos pelos testes utilizados. Demonstraram também ter adquirido e organizado todos os fonemas da língua (não fizeram uso de processos fonológicos e o inventário fonético das mesmas encontra-se completo).

$\mathrm{Na}$ avaliação pré-intervenção, foi constatado que os pré-escolares com DEL apresentaram comprometimento em todos os níveis do processamento da informação avaliados, comparado GC. É possível verificar essa constatação comparando as pontuações (médias) dos grupos na Tabela 1. Os valores de média referentes ao subteste memória sequencial auditiva do ITPA estão representados pela Idade Psicolinguística (IPL) obtida, em meses, 
pelos grupos. A média de idade cronológica das crianças com DEL é de 69,0 meses e a do GC é de 68,5 meses $(p=0,810)$. Analisando as médias na Tabela 1, é possível visualizar que a IPL média obtida pelo GC é compatível à idade cronológica média do grupo, enquanto a média obtida pelo grupo com DEL é bem inferior à respectiva idade cronológica.

As Tabelas 2, 3 e 4 permitem visualizar o desempenho dos pré-escolares com DEL nas duas avaliações a que foram submetidos, assim como comparar o desenvolvimento alcançado pelos mesmos após a intervenção. Ao fim do programa, todos apresentaram melhora na organização/produção fonológica (Tabela 4), assim como nos demais aspectos relacionados ao processamento fonológico avaliados e inseridos em intervenção - reconhecimento auditivo-fonético, consciência fonológica, acesso lexical, articulação e memória de curto prazo (Tabelas 2 e 3).

Tabela 1 - Comparação do desempenho psicolinguístico dos grupos na avaliação pré-intervenção

\begin{tabular}{lcc}
\hline Nível de processamento & Grupos & Média \\
\hline Reconhecimento & DEL & 23,0 \\
auditivo-fonético & GC & 33,0 \\
\hline \multirow{2}{*}{ Consciência fonológica } & DEL & 17,2 \\
& GC & 40,5 \\
\hline \multirow{2}{*}{ Acesso lexical } & DEL & 61,6 \\
& GC & 100,5 \\
\hline \multirow{2}{*}{ Memória de curto prazo } & DEL & 34,7 \\
& GC & 69,8 \\
\hline
\end{tabular}

Tabela 2 - Resultados das avaliações pré e pós-intervenção referentes aos níveis de processamento investigados

\begin{tabular}{lcccccccc}
\hline \multirow{2}{*}{ Nível de processamento } & \multicolumn{3}{c}{ Sujeito 1 } & \multicolumn{2}{c}{ Sujeito 2 } & \multicolumn{2}{c}{ Sujeito 3 } & \multicolumn{2}{c}{ Sujeito 4 } \\
\cline { 2 - 9 } & \multicolumn{2}{c}{ IC: 78} & \multicolumn{2}{c}{ IC: 60} & \multicolumn{2}{c}{ IC: 55} & IC: 83 \\
\cline { 2 - 9 } & Al & AF & Al & AF & Al & AF & Al & AF \\
\hline Reconhecimento Auditivo-fonético & 22 & 32 & 26 & 35 & 24 & 33 & 20 & 28 \\
Consciência Fonológica & 17 & 41 & 18 & 40 & 18 & 40 & 16 & 30 \\
Acesso Lexical & 63 & 72 & 85 & 99 & 50 & 86 & 49 & 72 \\
Memória de Curto Prazo & 45 & 56 & 34 & 56 & 30 & 56 & 30 & 39 \\
\hline
\end{tabular}

Legenda: Al- avaliação pré-intervenção; AF- avaliação pós-intervenção; IC- idade cronológica em meses

Tabela 3-Inventário fonético obtido pelos pré-escolares com DEL nas avaliações pré e pós-intervenção

\begin{tabular}{|c|c|c|}
\hline Sujeitos & $\mathrm{Al}$ & AF \\
\hline 01 & /p/;/b/;/t/;/d/; /s/;/m/; /n/; /n/; /l/; /R/; /r/ & $\begin{array}{c}\mathrm{p} / ; / \mathrm{b} / ; / \mathrm{t} / ; / \mathrm{d} / ; / \mathrm{f} / ; / \mathrm{v} / / \mathrm{s} / ; / \int / ; / \mathrm{j} / ; / \mathrm{m} / ; / \mathrm{n} / ; / \eta / \mathrm{m} \\
\text { /l/; /R/;/r/; /l/ } \\
(16)\end{array}$ \\
\hline 02 & $\begin{array}{c}/ \mathrm{p} / ; / \mathrm{b} / ; / \mathrm{t} / ; / \mathrm{d} / ; / \mathrm{f} / ; / \mathrm{m} / ; / \mathrm{n} / ; \eta \mathrm{\eta} / ; / \mathrm{R} / \\
(9)\end{array}$ & $\begin{array}{c}/ \mathrm{p} / ; / \mathrm{b} / ; / \mathrm{t} / ; / \mathrm{d} / ; / \mathrm{f} / ; / \mathrm{v} / ; / \mathrm{s} / ; / \mathrm{z} / ; / \mathrm{m} / ; / \mathrm{n} / ; / \mathrm{l} / ; / / / ; \\
/ \mathrm{R} / ; / \mathrm{r} / \\
(14)\end{array}$ \\
\hline 03 & $\begin{array}{c}\text { /p/; /b/;tt/; /k/; /s/; / J /; /j/; /m/; /n/; /n/; /l/; /R/ } \\
(12)\end{array}$ & $\begin{array}{c}\mathrm{p} / ; / \mathrm{b} / ; / \mathrm{t} / ; / \mathrm{d} / ; / \mathrm{k} / ; / \mathrm{g} / ; / \mathrm{f} / ; / \mathrm{v} / ; / \mathrm{s} / ; / \mathrm{z} / ; / \int \mathrm{J} / ; / \mathrm{j} / \\
/ \mathrm{m} / ; / \mathrm{h} / ; / \mathrm{l} / ; / \mathrm{l} / ; / \mathrm{R} / \\
(17)\end{array}$ \\
\hline 04 & $\begin{array}{c}\mathrm{p} / ; / \mathrm{b} / ; / \mathrm{t} / ; / \mathrm{d} / ; / \mathrm{f} / ; / \mathrm{v} / ; / \mathrm{s} / ; / \mathrm{z} / ; / \int / ; / \mathrm{m} / ; / \mathrm{n} / ; / \mathrm{n} / \\
/ \mathrm{l} / ; / \mathrm{R} / ; / \mathrm{r} / \\
(15)\end{array}$ & $\begin{array}{c}\mathrm{p} / ; / \mathrm{b} / ; / \mathrm{t} / ; / \mathrm{d} / ; / \mathrm{k} / ; / \mathrm{g} / ; / \mathrm{f} / ; / \mathrm{v} / ; / \mathrm{s} / ; / \mathrm{z} / ; / \int / ; / \mathrm{r} / \\
/ \mathrm{m} / ; / \mathrm{n} / ; / \mathrm{n} / ; / \mathrm{l} / ; / \mathrm{R} / \\
(17)\end{array}$ \\
\hline
\end{tabular}

Legenda: Al- avaliação pré-intervenção; AF- avaliação pós-intervenção 
Tabela 4 - Processos fonológicos utilizados pelos pré-escolares com DEL nas avaliações pré e pós-intervenção

\begin{tabular}{|c|c|c|}
\hline Sujeitos & Avaliação pré-intervenção & Avaliação pós-intervenção \\
\hline 01 & $\begin{array}{c}\text { F.Vel.-100\% } \\
\text { P.Fric.- } 86,9 \% \\
\text { E.Plo- } 44,4 \% \\
\text { S.Liq.-27,2\% } \\
\text { S.E.Cons - 62,5\% }\end{array}$ & $\begin{array}{c}\text { P.Fric.-34,7\% } \\
\text { E.Plo.-28,5\% } \\
\text { S.Liq. }-27,2 \% \\
\text { S.E.Cons- } 62,5 \%\end{array}$ \\
\hline 02 & $\begin{array}{c}\text { F.Vel.- } 33,3 \% \\
\text { S.Liq.-72,7\% } \\
\text { S.C.Fin.-100\% } \\
\text { F.Pal.-80\% } \\
\text { S.E.Cons.-100\% } \\
\end{array}$ & $\begin{array}{l}\text { S.E.Cons-50\% } \\
\text { S.C.Fin- } 80 \% \\
\text { S.Liq.-50\% }\end{array}$ \\
\hline 03 & $\begin{array}{c}\text { S. E.Con.-100\% } \\
\text { S.C.Fin.-100\% } \\
\text { F.Vel.-33,3\% } \\
\text { S. Liq.- }-72,7 \% \\
\text { F. Pal.-80\% } \\
\end{array}$ & $\begin{array}{l}\text { S.E. Cons. }-60 \% \\
\text { S.C. Fin. }-80 \% \\
\text { S.Liq. }-57 \%\end{array}$ \\
\hline 04 & $\begin{array}{l}\text { S.E.Con.-100\% } \\
\text { F.Vel.-77,7\% } \\
\text { S.Liq.-36,3\% } \\
\text { F.Pal.-40,0\% }\end{array}$ & $\begin{array}{l}\text { S.Enc.-87,5\% } \\
\text { S.Liq. }-27,2 \%\end{array}$ \\
\hline
\end{tabular}

Legenda: F.Vel. - frontalização de velar; P.Fric. - plosivação de fricativas; S.E.Com - simplificação de encontro consonantal; E.Plos ensurdecimento de plosivas; S.Liq.- simplificação de líquidas; S.C.Final- simplificação de consoante final; F.Vel.- frontalização de velar

\section{DISCUSSÃO}

Este trabalho foi desenvolvido com o objetivo de verificar a eficácia da intervenção fonoaudiológica, com base em um Modelo Psicolínguístico, no desenvolvimento fonológico de crianças com DEL. Para tal, alguns cuidados foram tomados, no sentido de garantir a coerência entre a prática implementada neste estudo e os princípios dos Modelos Psicolinguísticos.

Antes de qualquer procedimento ser realizado, escolheu-se o Modelo que guiaria o processo de avaliação e intervenção. A partir da escolha do mesmo, foram determinadas as provas ou testes que seriam utilizados dentro do processo de avaliação e que permitiriam que este fosse desenvolvido de uma forma detalhada, propiciando a descrição dos processos diretamente envolvidos com a linguagem - mais especificamente com o sistema fonológico - assim como a interação entre eles e os processos cognitivos subjacentes ${ }^{7}$.

Os resultados da avaliação pré-intervenção demonstraram que as crianças com DEL deste estudo apresentaram dificuldades relacionadas ao desenvolvimento do sistema fonológico, manifestadas, principalmente, pelo uso produtivo de processos fonológicos não esperados para a idade na fala espontânea. A partir dessa constatação, algumas questões foram levantadas e os referidos testes de avaliação foram utilizados na tentativa de formular hipóteses a respeito da natureza das dificuldades manifestadas: 1. será que as crianças do estudo são capazes de realizar a discriminação entre os sons da fala?; 2. as representações fonológicas das palavras são precisas?; 3. elas são conscientes da estrutura fonológica das palavras?; 4. são capazes de manipular tais representações? 5 . são capazes de selecionar e acessar no léxico mental as representações fonológicas com o intuito de produção?; 6. são capazes de articular as palavras com precisão?; 7. existe um comprometimento da capacidade de memória de curto prazo que possa estar impedindo o desenvolvimento fonológico adequado?; 8. as dificuldades fonológicas enfrentadas por essas crianças são decorrentes de falhas no processamento receptivo e/ou expressivo da informação?; 9. são decorrentes de falhas no processamento central e/ou periférico?; 10 . o desenvolvimento de cada um desses aspectos citados repercutirá na organização fonológica?; 11. o desenvolvimento obtido na produção fonológica resultará no desenvolvimento da consciência fonológica?

Considerando o nível receptivo do processamento, as crianças demonstraram que discriminam auditivamente mal pares de palavras (Tabela 1), evidenciando uma ruptura num nível mais 
periférico do processamento receptivo. De acordo com esquema Psicolinguístico proposto neste estudo, os déficits dos níveis mais periféricos condicionam amplamente o desenvolvimento e/ou o funcionamento dos níveis mais centrais. Assim, o prejuízo do nível gnósico (integração/percepçãoauditiva), com uma audição intacta, repercute sobre a decodificação da mensagem não permitindo que o nível terciário do processamento ocorra adequadamente ${ }^{1}$.

A dificuldade de realizar o reconhecimento auditivo-fonético de palavras foi levantada como uma das possíveis causas para as dificuldades relacionadas ao desenvolvimento fonológico dos pré-escolares. Portanto, durante o processo de intervenção foram desenvolvidas estratégias voltadas para o treino da discriminação auditiva. $\mathrm{Na}$ Tabela 2 é possível observar que um desenvolvimento considerável desta habilidade foi obtido por todos os pré-escolares. Este resultado sugere que para aprimorar esta habilidade, um treino específico, com enfoque neste nível da análise da informação, é necessário. Reflete a efetividade das estratégias implementadas neste estudo para desenvolver a capacidade de discriminação auditiva das crianças. E confirma que a dificuldade de discriminação auditiva encontra-se intimamente relacionada às dificuldades de recepção e organização do sistema fonológico das crianças com DEL, visto o desenvolvimento também obtido na avaliação desse subsistema linguístico, ao fim do processo de intervenção ${ }^{7,10-12}$.

As crianças com DEL também apresentaram comprometimento na realização de tarefas metafonológicas, na avaliação pré-intervenção, sinalizando a imprecisão das representações fonológicas, dificuldades em manipulá-las e a não emergência de consciência sobre a estrutura fonológica das palavras. Mais especificamente, refletem o comprometimento do nível central do processamento fonológico, tanto receptivo como expressivo. Ao fim do processo de intervenção, obtiveram um desenvolvimento considerável na realização das referidas habilidades (Tabela 2), assim como na produção fonológica, confirmando que a presença de dificuldades fonológicas severas não restringe o acesso dessas crianças aos benefícios da intervenção metafonológica ${ }^{13-15}$.

Também é possível afirmar que as estratégias selecionadas e implementadas foram ao encontro das necessidades dos pacientes, o que atesta que a hipótese inicial levantada foi condizente com as habilidades e deficiências das crianças e confirma a necessidade de implementação de estratégias individuais para $\mathrm{o}$ desenvolvimento metafonológico ${ }^{15}$. Isso porque as atividades de consciência fonológica, mesmo que inseridas dentro de uma mesma categoria, requerem diferentes demandas de processamento, em diferentes níveis. Algumas atividades envolvem o processamento no nível receptivo e/ou expressivo, outras exigem que a criança acesse o léxico mental ou podem utilizar estímulos não-familiares, enquanto outras exigem a participação da memória de trabalho e a manipulação das unidades fonológicas ${ }^{2}$.

Como se sabe, os processos de desenvolvimento cognitivo e metalinguístico ocorrem de forma conjunta e inter-relacionada. A partir da estruturação do seu sistema fonológico e da possibilidade de produzir corretamente os sons da fala, a criança mostra-se apta a realizar algumas tarefas, as quais serão aprimoradas a partir da aquisição do código escrito ${ }^{14}$. Assim, se a criança apresenta dificuldades na produção fonológica, dificilmente poderá fazer reflexões sobre esse componente, uma vez que, para realizar as tarefas metafonológicas é necessário que além de refletir, a criança acesse as representações fonológicas ao nível de fonema, sílaba, palavras ou frases. Logo, se essas representações não se encontram bem estabelecidas, o acesso às estruturas requisitadas fica comprometido.

Assim sendo, pode ser também que o desenvolvimento obtido na produção fonológica tenha repercutido no desempenho dos pré-escolares em tarefas metalinguísticas ao fim do programa de intervenção, coincidindo com os estudos sobre o tema que abordam as relações entre desenvolvimento linguístico e metalinguístico ${ }^{13,14}$.

Além de ser necessário que as operações e processamentos linguísticos fonológicos, semânticos, lexicais ocorram de forma adequada, o desempenho metafonológico também se encontra relacionado aos aspectos cognitivos não linguísticos, como a memória de trabalho ${ }^{2}$. Em atividades metafonológicas é necessário que a criança retenha os estímulos por um breve período de tempo enquanto faz um julgamento a respeito da estrutura fonológica das palavras. Como se sabe, as crianças com DEL são deficitárias em tarefas de memória operacional, que envolvem o armazenamento de um estímulo verbal, enquanto a informação está sendo processada ${ }^{4,5}$. Como os pré-escolares do estudo também apresentaram desempenho aquém do esperado na avaliação da memória auditiva de curto prazo (Tabela 1), é possível que o déficit nessa habilidade tenha contribuído para um pobre desempenho metafonológico, relação esta também verificada em estudos anteriormente realizados ${ }^{2}$.

Lembrando que a memória de trabalho é um sistema de armazenamento da informação de curto prazo, conceituada como arquivo dinâmico de informações, formada por diversos componentes que 
selecionam e manipulam as informações verbais, visuais e espaciais, durante alguns segundos, antes de transmiti-los à memória de longo prazo e a outros sistemas cognitivos. Sendo assim, a limitação na memória de curto prazo manifestada pelas crianças do estudo, pode estar também relacionadas ao comprometimento em aprender a forma fonológica das palavras, prejudicando o aprendizado de palavras novas, já que esse tipo de memória trabalha como um mediador para a construção das representações fonológicas permanentes na memória de longo prazo ${ }^{3,4}$.

Alguns estudos anteriormente realizados encontraram, inclusive, relação positiva entre a capacidade da memória de trabalho com o grau de severidade do distúrbio fonológico, o que segundo os autores, permite aceitar a ideia de que a memória fonológica está relacionada com a produção da fala, mais especificamente, com a escolha dos fonemas para a produção das palavras ${ }^{3}$.

No que diz respeito ao processo de intervenção junto a essa atividade, a literatura defendeu que as intervenções mais bem sucedidas são aquelas dirigidas às influências bidirecionais entre linguagem-memória, enfatizando, para pré-escolares, atividades que destaquem a estrutura fonológica da língua, de modo a desenvolver as habilidades de codificação e representação fonológica ${ }^{2}$. Os resultados da avaliação pós-intervenção (Tabela 2) demonstram que os pré-escolares conseguiram melhorar seu desempenho em atividade de memória de curto prazo, o que certamente favoreceu o processamento geral, considerando os aspectos relacionados com a recepção e também com a produção fonológica (análise fonológica, acesso lexical e produção fonológica).

No nível expressivo, a melhora ou não na repetição de algumas palavras indicou dificuldades na recuperação da informação fonológica no léxico mental, dificuldades ao nível de representação e/ou na produção articulatória dos fonemas ${ }^{7}$. Ao fim do programa, constatou-se que houve uma expansão do inventário fonético de todos os pré-escolares (Tabela 3).

Ao apresentar um esquema representativo do Modelo Psicolinguístico, o autor chamou a atenção dos clínicos para a disposição vertical do Modelo e à hierarquização dos níveis. Relatou que efetivamente, os déficits nos níveis mais periféricos condicionam amplamente o desenvolvimento e/ou funcionamento dos níveis mais centrais. Mais especificamente deixou claro que na vertente expressiva, um distúrbio importante da motricidade, no nível primário, pode impedir qualquer expressão inteligível ${ }^{~}$. Diante dessa colocação, o programa de intervenção explicitamente utilizou estratégias como exercícios articulatórios, implantação de fonemas com pistas táteis e cinestésicas, como proposto ${ }^{1,7}$, visando a expansão do inventário fonético dos sujeitos envolvidos, o que resultou também em benefícios na produção fonológica.

Em relação à produção/organização fonológica, pôde-se observar que houve uma diminuição do número de processos fonológicos utilizados pelos os sujeitos no final de todo o processo de intervenção, acarretando em melhoria da inteligibilidade de fala (Tabela 4). Esse resultado concorda com outros já realizados. Nestes, diferentes Modelos de intervenção com enfoque no processamento da informação, foram aplicados no tratamento de crianças com distúrbio fonológico de naturezas distintas. Os autores concluíram que o tratamento que enfoca o(s) nível(s) de processamento da fala subjacente(s) ao distúrbio resulta numa eficiente mudança no sistema fonológico das mesmas ${ }^{6,12}$.

Os resultados apresentados e discutidos neste estudo sugerem que a(s) hipótese(s) levantada(a) sobre a natureza das dificuldades fonológicas encontradas foram condizentes com as habilidades e deficiências das crianças, que a intervenção abordou efetivamente os aspectos comprometidos e que, quando bem empregado, o Modelo Psicolinguístico constitui uma ferramenta eficaz no tratamento das dificuldades fonológicas de crianças com DEL, cujas dificuldades encontram-se situadas nos níveis de análise e produção linguística investigados neste trabalho ${ }^{9}$.

Contudo, é extremamente importante ressaltar que apesar da melhora observada no desempenho psicolinguístico dos pré-escolares, o rendimento em algumas habilidades ainda continua aquém do esperado para a faixa etária. Nesse caso, será dada continuidade ao processo terapêutico visando promover o desenvolvimento dos referidos aspectos relacionados ao sistema fonológico.

Os resultados apresentados também devem ser interpretados cautelosamente, em decorrência da amostra restrita do estudo, que não permitiu uma análise estatística, impossibilitando a generalização dos dados. Entretanto, isso não elimina a possibilidade desse procedimento de intervenção ser utilizado em futuras investigações, até mesmo com crianças que apresentam dificuldades de linguagem de outras naturezas.

Diante de todas as informações apresentadas, acredita-se que pesquisas com Programas de intervenção, apesar de trabalhosas e arriscadas, em função do grande número de variáveis a serem controladas e por se tratarem de estudos longitudinais, são fundamentais, não só para a verificação da eficácia do(s) procedimento(s) de interesse, mas também para o entendimento deste universo 
que são os mecanismos envolvidos numa função tão complexa e especificamente humana, como é a linguagem.

\section{CONCLUSÃO}

A utilização do Modelo Psicolinguístico demonstrou ser efetiva no tratamento das dificuldades fonológicas das crianças com DEL deste estudo. O desenvolvimento dos aspectos psicolinguísticos previamente comprometidos também comprova que o uso de procedimentos de avaliação adequados aos diversos níveis do processamento torna possível a compreensão da natureza dos transtornos de linguagem e a programação de estratégias de intervenção mais efetivas para as dificuldades fonológicas de crianças com DEL.

\begin{abstract}
Background: phonologic intervention for children with Specific Language Impairment, within a Psycholinguistic Model. Procedures: this study involved four pre-school children, aging between 48 to 83 months, that had not been previously submitted to intervention. The psycholinguistic assessment was carried pre and post-intervention, through several instruments that evaluate diverse levels of information processing: auditory-phonetic recognition, lexical access, phonological working memory, phonological awareness, articulation, phonological production. The intervention took 4 months. Results: preschool children showed difficulties in diverse evaluated processing information levels(preintervention), showing that the difficulties on phonological organization were related to problems in the receptive and expressive processing. To the end of the Program, all had presented improvement in the phonological performance. Conclusion: the use of evaluation instruments being suitable to diverse phonological processing levels propitiates the understanding on the nature of language impairments and the programming of more effective intervention strategies.
\end{abstract}

KEYWORDS: Language Development Disorders; Language Tests; Language Therapy

\section{REFERÊNCIAS}

1. Chevrie-Muller C. Exploração da linguagem oral. In: Chevrie-Muller C, Narbona J, editors. A linguagem da criança: aspectos normais e patológicos. 2. ed. Porto Alegre: Artmed; 2005. p. 101-33.

2. Montgomery JW. Working memory and comprehension in children with specific language impairment: what we know so far. J Commun Disord. 2003 May-June; 36(3):221-31.

3. Linassi LZ, Keske-Soares M, Mota HB. Habilidades de memória de trabalho e o grau de severidade do desvio fonológico. Pró-Fono. 2005 set-dez; 17(3):383-92.

4. Archibald LMD, Gathercole SE. Short-term and working memory in specific language impairment. Int J Lang Commun Disord. 2006; 41(6):675-93.

5. Marton K, Schwartz RG. Working memory capacity and language processes in children with specific language impairment. J Speech Lang Hear Res. 2003 Oct; 46(5):1138-53.

6. Crosbie S, Holm A, Dodd A. Intervention for children with severe speech disorder: a comparison of two approaches. Int $\mathrm{J}$ Lang Commun Disord. 2005; 40(4):467-91.
7. Cervera-Mérida JF, Ygual-Fernández A. Intervención logopédica en los trastornos fonológicos desde el paradigma psicolingüístico del procesamiento del habla. Rev Neurol. 2003; 36(Supl1):S39-S53.

8. Pascoe M, Stackhouse J, Wells B. Phonological therapy within a psycholinguistic framework: promoting change in a child with persisting speech difficulties. Research report. Int J Lang Commun Disord. 2005; 40(2):189-220.

9. Baker E, Croot K, McLeod S, Paul R. Psycholinguistic models of speech development and their application to clinical practice. J Speech Lang Hear Res. 2001 Jun; 44(3):685-702.

10. Wolfe V, Presley C, Mesaris J. The importance of sound identification training in phonological intervention. Am J Speech Lang Pathol. 2003 Aug; 12(3):282-8.

11. Rvachew S, Nowak M, Cloutier G. Effect of phonemic perception training on the speech production and phonological awareness skills of children with expressive phonological delay. Am J Speech Lang Pathol. 2004 Aug; 13(3):250-63. 
12. Joffe V, Pring T. Children with phonological problems: a survey of clinical practice. Int $\mathrm{J}$ Lang Commun Disord. 2008; 43(2):154-64.

13. Segers E, Verhoeven L. Computer-supported phonological awareness intervention forkindergarten children with specific language impairment. Lang Speech Hear Serv Sch. 2004 Jul; 35(3):229-39.

14. Ortiz ZB, Tirapegui CJC. La conciencia fonológica en niños con trastorno específico de lenguaje. Rev Logop Foniatr Audiol. 2004; 24(4):156-63.

15. Denne M, Langdown T, Pring T, Roy P. Treating children with expressive phonological disorders: does phonological awareness therapy work in the clinic? Int J Lang Commun Disord. 2005 Oct-Dec; 40(4):493-504.

16. Serrano B. Informática na fonoaudiologia: teste de discriminação auditiva. Disponível em: URL: http://www.informaticanafonoaudiologia.hpg. ig.com.br/004discriminacaoauditiva.htm.
17. Alvarez AM, Carvalho IAM, Caetano AL. Perfil de habilidades fonológicas. 2. ed. São Paulo: Via Lettera; 2004. 12p.

18. Béfi-Lopes DM. Vocabulário. In: Andrade CRF, Béfi-Lopes DM, Fernandes FDM, Wertzner HF. $A B F W$ : teste de linguagem infantil nas áreas de fonologia, vocabulário, fluência e pragmática. 2. ed. Carapicuíba: Pró-Fono; 2004. p. 41-59.

19. Wertzner HF. Fonologia. In: Andrade CRF, Béfi-Lopes DM, Fernandes FDM, Wertzner HF. $A B F W$ : teste de linguagem infantil nas áreas de fonologia, vocabulário, fluência e pragmática. 2. ed. Carapicuíba: Pró-Fono; 2004. p.1-40.

20. Bogossian MA, Santos MJ. Teste Illinois de Habilidades Psicolinguísticas. Rio de Janeiro: EMPSI; 1977.

DOI: 10.1590/S1516-18462009005000057

RECEBIDO EM: 26/05/2008

ACEITO EM: 10/09/2009

Endereço para correspondência:

Dáphine Luciana Costa Gahyva

Av. Leopoldino de Oliveira, 2371 ap. 801

Uberaba - MG

CEP: 38015-000

E-mail: daphiusp@yahoo.com 